\title{
Influence of the Surface Mechanical Treatment on the Photothermal Piezoelectric Spectra of $\mathrm{ZnSe}$ Crystals
}

\author{
J. Zakrzewski • M. Maliński · K. Strzałkowski
}

Received: 10 November 2011 / Accepted: 11 July 2012 / Published online: 25 July 2012

(C) The Author(s) 2012. This article is published with open access at Springerlink.com

\begin{abstract}
This paper presents experimental and theoretical piezoelectric photoacoustic spectra of ZnSe samples after different surface treatments: grinding, polishing, and etching. The modification of the Jackson and Amer theory, presented in the paper, enabled numerical interpretation of the spectra in the model of a mechanically damaged surface layer. The nature of the characteristic peak observed at $E=2.65 \mathrm{eV}$ below the energy gap is explained as caused by the Urbach absorption tail and the damaged surface layer of a sample. The correlation between the microhardness of the $\mathrm{Zn}_{1-x} \mathrm{Be}_{x}$ Se and $\mathrm{ZnSe}$ crystals and the thickness of the mechanically damaged surface layer was observed.
\end{abstract}

Keywords Piezoelectric photothermal spectroscopy · II-VI Semiconductors • Surface quality $\cdot \mathrm{ZnSe}$

\section{Introduction}

Photothermal methods [1] represent a useful tool for measurements of the optical, thermal, and recombination parameters of semiconductors [2-7]. The significance of thermal phenomena becomes more and more important because of the problem of energy dissipation in miniaturized semiconducting devices. The piezoelectric photothermal (PZE) technique developed by Jackson and Amer (J-A) [8] and by Blonskij et al. [9]

J. Zakrzewski (凶) $\cdot$ K. Strzałkowski

Instytut Fizyki, Uniwersytet Mikołaja Kopernika, ul. Grudziądzka 5/7, 87-100 Toruń, Poland e-mail: jzakrzew@ fizyka.umk.pl

\section{Maliński}

Department of Electronics and Computer Science, Technical University of Koszalin,

2 Śniadeckich St., 75-328 Koszalin, Poland 
was successfully used to investigate the properties of A2B6 and mixed crystals [1012]. In the course of the studies, it turned out that the surface preparation process of samples strongly influenced the photoacoustic spectra [13]. It could be emphasized that surface effects are easy to be observed in the case of piezoelectric detection in contrast to the typical, microphone detection measurements.

The piezoelectric method takes advantage of processes that occur in solid-state samples heated by a periodically modulated beam of light. As a result of periodical heating, a spatial and temporal temperature field arises in the sample. The dependence of the amplitude and phase spectra of the piezoelectric signals on the value of the optical absorption coefficient is strongly nonlinear. Additional difficulty arises from the fact that analyzed crystals are often not perfect, what results in the necessity of the development of special numerical models for interpretation of the spectra. For proper interpretation of amplitude and phase spectra, one needs to know or simulate the temperature spatial distribution in the sample and the absorption dependence on energy incident on the sample. J-A and Blonskij's theories deal with the perfect sample, and some modifications are necessary to obtain the photothermal piezoelectric spectra of a real sample. They could be done due to the effects of the shape of the sample, inhomogeneity of the material, mechanically damaged surface, or the presence of defect states located at the surfaces [10-13].

Wide gap II-VI compounds are still the subject of intense research, mainly because of their application in bandgap engineering, interband and intersubband transitions, and dilute semiconductor materials and devices. Among others, ZnSe appears promising as a wide-bandgap semiconductor for applications in high power/high-frequency devices, blue and ultraviolet light-emitting devices, photodetectors, and chemically stable substrates for epitaxial growth of various materials. For mixed crystals, based on $\mathrm{ZnSe}$, it is possible to change electronic properties, lattice parameters, and bandgap energies by adjusting the composition of the crystal. The change of the bandgap energy gives the desired optical properties and emission in the entire visible and uv ranges of the spectrum.

From the point of view of the quality of optoelectronic devices based on $\mathrm{ZnSe}$ crystals, the quality of the surface of the samples is of great importance. This paper shows results of piezoelectric spectral studies of $\mathrm{ZnSe}$ samples, indicating that this measuring method can bring useful information about the quality of the samples after different surface treatments.

The experimental effect that is analyzed and interpreted numerically in this paper is a much smaller value of the amplitude of the piezoelectric signal in the band-toband absorption region in proportion to the value in the Urbach absorption tail than is expected from model computations of the piezoelectric photothermal amplitude spectra of ZnSe samples. This experimental effect was also presented in other papers [11,14-16].

\section{Sample Preparation}

All the samples were grown from high-purity powder with the high-pressure Bridgman method. The crystal rods were cut into about $1 \mathrm{~mm}$ to $1.2 \mathrm{~mm}$ thick samples which 
were first ground using grinding powder (diameter of $10 \mu \mathrm{m}$ ). Some of them were then polished with diamond paste $(1 \mu \mathrm{m})$ and finally chemically etched. A solution of $\mathrm{H}_{2} \mathrm{SO}_{4}(96 \%), \mathrm{K}_{2} \mathrm{Cr}_{2} \mathrm{O}_{7}$, and water was used for etching the samples. After etching, the samples were rinsed in distilled water and then put for a few seconds in boiling $\mathrm{NaOH}$. Then the samples were rinsed again in cold water, next in boiling distilled water, and finally in ethyl alcohol. Some samples were measured after cutting, etching in $\mathrm{HCl}$, and rinsing in water.

\section{Photoacoustic Signal Generation}

The basic information needed for the proper interpretation of the piezoelectric spectratemperature spatial distribution in the sample - was given by Jackson and Amer (J-A) [8] and Blonskij et al. [9]. The temperature distribution in the sample is the basis for the finding of the theoretical amplitude and phase photoacoustic spectra [10]. These formulae are as follows:

$$
\begin{aligned}
& T(x)=\frac{\left(1-R_{\mathrm{opt}}\right) \beta I_{0}}{2 \lambda \sigma(1-R \exp (-2 \sigma l))}[M(x)+N(x)] \\
& M(x)=\frac{[\exp (\sigma x)+\exp (-\sigma x)][\exp ((-\sigma-\beta) x)-\exp ((-\sigma-\beta) l)]}{\beta+\sigma} \\
& +\frac{R \exp (-2 \sigma l)[\exp (\sigma x)+\exp (-\sigma x)][\exp ((\sigma-\beta) x)-\exp ((\sigma-\beta) l)]}{\beta-\sigma} \\
& N(x)=\frac{[\exp (-\sigma x)+R \exp (-2 \sigma l+\sigma x)][1-\exp ((-\sigma-\beta) x)]}{\beta+\sigma} \\
& +\frac{[\exp (-\sigma x)+R \exp (-2 \sigma l+\sigma x)][1-\exp ((\sigma-\beta) x)]}{\beta-\sigma}
\end{aligned}
$$

where $l$ is the thickness of a sample, $x$ is the spatial coordinate, $\beta(E)$ is the optical absorption coefficient spectrum, $\sigma^{2}=\mathrm{i} \omega / \alpha, \alpha$ is the thermal diffusivity, $\omega=2 \pi f, f$ is the modulation frequency, $\lambda$ is the thermal conductivity of the sample, and $R_{\mathrm{opt}}$ is the optical reflection coefficient defined as the ratio of the intensity of light reflected from the surface of the sample to the intensity of light illuminating the surface.

$R$ is the thermal reflection coefficient defined as the ratio of the amplitude of the thermal wave reaching the surface of the sample to the amplitude of the thermal wave reflected from the surface.

In piezoelectric detection, this distribution introduces the stress and strain and causes the thermal expansion and bending of the sample. These effects are called piston and drum. In piezoelectric experiments the sum or difference of these two effects is observed simultaneously depending on the applied experimental configuration, which are called front and rear configurations [10]. For that reason, different shapes of amplitude and phase spectra in the case of front and rear detections are observed.

For the rear experimental configuration, the amplitude and phase piezoelectric spectra can be computed with the expression given by the one-dimensional (1D) formula, which is a J-A expression[8]: 

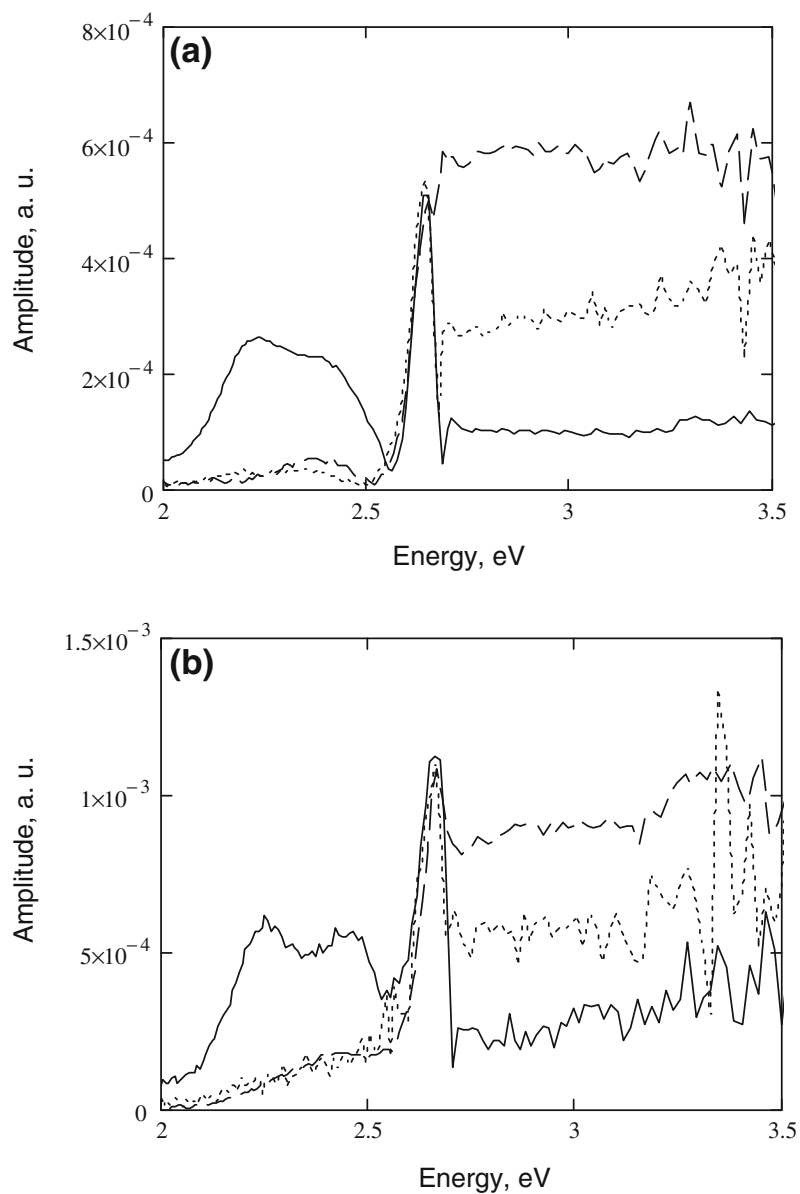

Fig. 1 Amplitude piezoelectric spectra of ground solid lines, polished dotted lines, and etched dashed lines of two samples of ZnSe: (a) sample 1 and (b) sample 2. Thickness of the samples $l=0.11 \mathrm{~cm}$, frequency of modulation $f=126 \mathrm{~Hz}$, thermal diffusivity $\alpha=0.05 \mathrm{~cm}^{2} \cdot \mathrm{s}^{-1}$

$$
S \cong k\left(\frac{1}{l} \int_{0}^{l} T(x) \mathrm{d} x-\frac{6}{l^{2}} \int_{0}^{l}\left(\frac{l}{2}-x\right) T(x) \mathrm{d} x\right)
$$

$k$ is the resilience coefficient of the sample-Young's modulus. It is assumed in this one-layer model that its value is identical for the whole thickness of the sample.

The modifications introduced to the temperature distributions allow interpretation of the spectra of nonideal crystal samples. So far, these modifications covered the inhomogeneity of the material and surface defects [10]. The presence of the defects introduces additional features to the piezoelectric spectra: maxima in the amplitude spectra, in the region of energies smaller than the energy gap of the sample, accompanied by appropriate changes in the phase spectra. Recently, a quite new behavior in the 
Fig. 2 (a) Simulation of the amplitude of the photoacoustic spectrum of the sample with a damaged layer of the thickness: $d=0.005 \mathrm{~cm}$ (solid line), $d=0.013 \mathrm{~cm}$ (dotted line), $d=0.02 \mathrm{~cm}$ (dashed line). (b) Simulation of the phase of the photoacoustic spectrum of the sample with a damaged layer of the thickness: $d=0.004 \mathrm{~cm}$, (solid line), $d=0.013 \mathrm{~cm}$ (dotted line), $d=0.02 \mathrm{~cm}$ (dashed line)
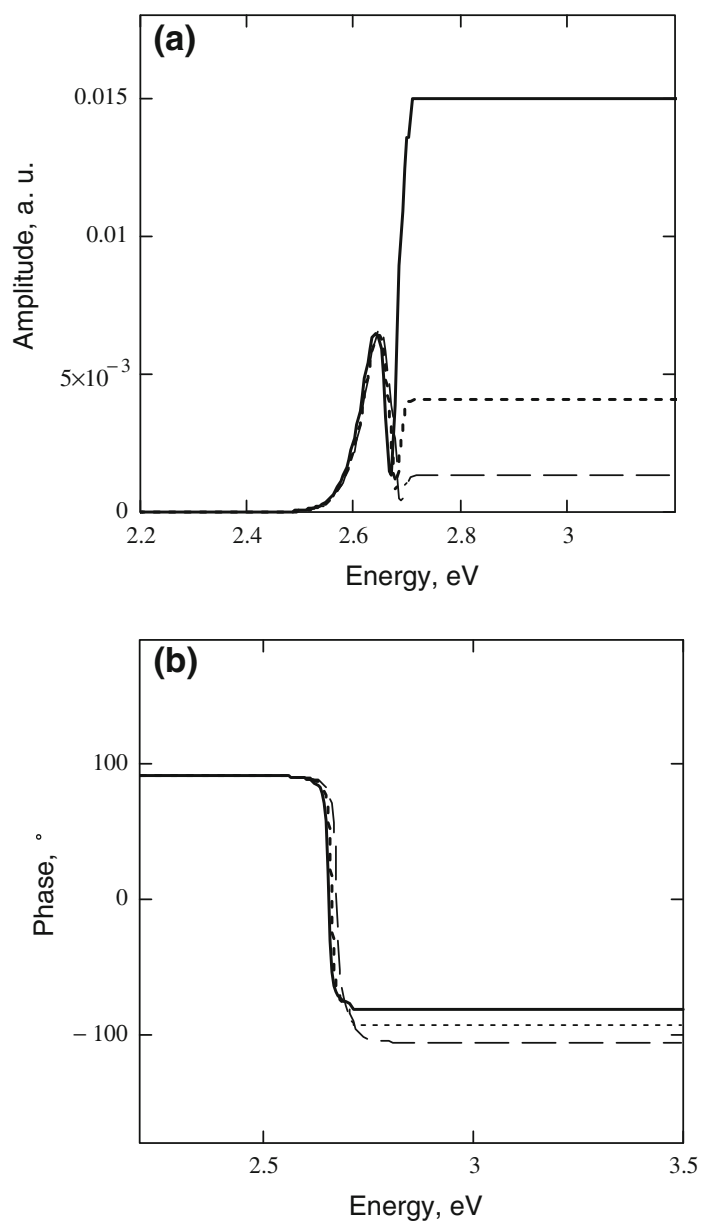

character of the piezoelectric spectra has been observed for ZnSe samples, measured after a different procedure of surface preparations. The existing piezoelectric photoacoustic models do not allow proper interpretation of that kind of behavior, and some modifications of the expression for the piezoelectric signal (Eq. 4) are necessary.

\section{Results}

Figures 1a and b present the experimental amplitude piezoelectric spectra of ground (solid line), polished (dotted line), and etched (dashed line) samples of $\mathrm{ZnSe}$.

Two additional maxima in the sub-bandgap region, not expected in an ideal J-A model, are present. The maximum at the energy $E=2.65 \mathrm{eV}$ dominates in the spectra of ground samples. It is the result of the Urbach tail of the optical absorption spectrum. The maxima at the energy $E=2.22 \mathrm{eV}$ and $E=2.40 \mathrm{eV}$ are caused by the surface 
Fig. 3 Fit of the theoretical curve to the experimental piezoelectric spectrum of the ground $\mathrm{ZnSe}$ sample 2. Solid line theoretical curve, circles experimental data of sample 2 . Parameters: $l=0.11 \mathrm{~cm}, f=$ $126 \mathrm{~Hz}, \alpha=0.05 \mathrm{~cm}^{2} \cdot \mathrm{s}^{-1}$, thickness of a damaged layer $d=0.0185 \mathrm{~cm}$
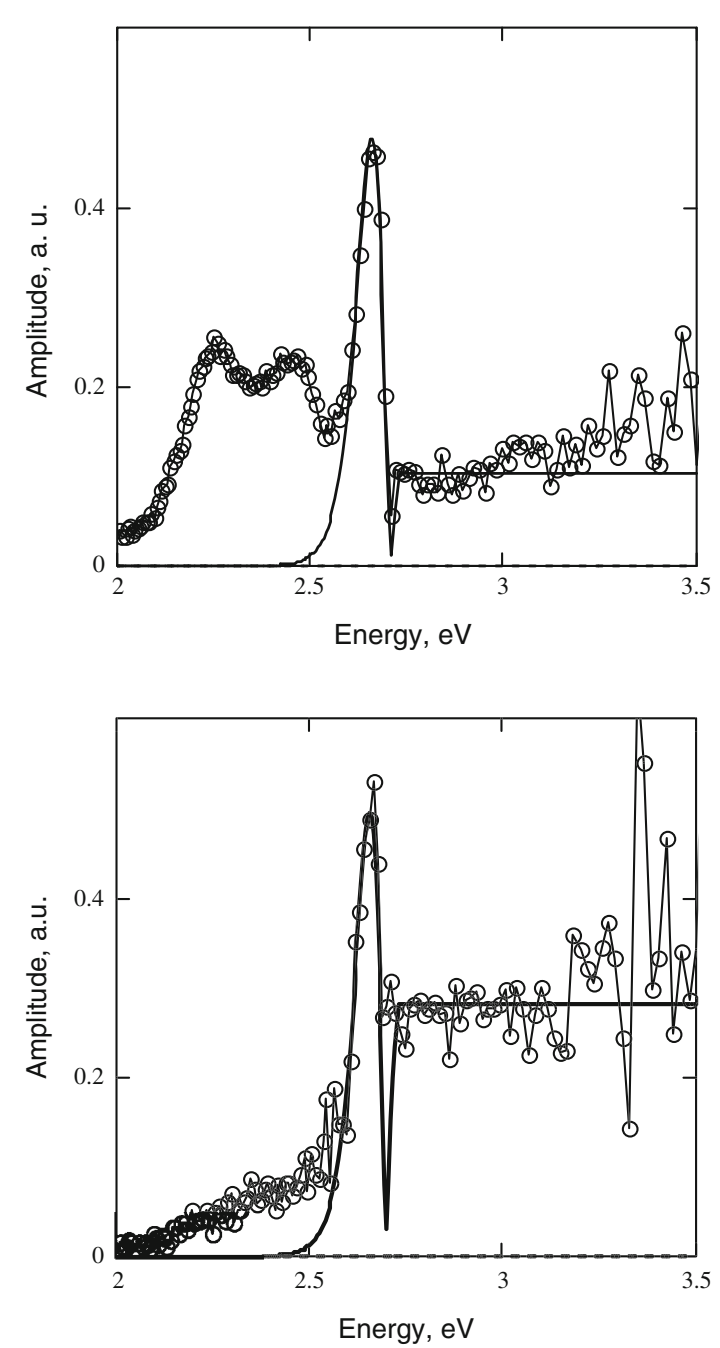

Fig. 4 Fit of the theoretical curve to the experimental piezoelectric spectrum of the polished ZnSe sample 2. Solid line theoretical curve, circles experimental data of sample 2 . Parameters: $l=0.11 \mathrm{~cm}, f=$ $126 \mathrm{~Hz}, \alpha=0.05 \mathrm{~cm}^{2} \cdot \mathrm{s}^{-1}$, thickness of a damaged layer $d=0.016 \mathrm{~cm}$

states of the sample depending on the surface preparation. These bands dominate in the ground samples and are strongly reduced by the polishing and etching procedures.

These spectra exhibit characteristic features: for energies of photons larger than the energy gap of the crystal $\left(E_{\mathrm{g}}=2.72 \mathrm{eV}\right)$, the amplitudes of the piezoelectric signal are the smallest for ground samples, larger for polished samples, and largest for the etched samples while the peak at $E=2.65 \mathrm{eV}$ remains unchanged. Thus, the effect of improvement of the piezoelectric spectra is clearly visible as a function of the quality of the surface of the samples.

For interpretation of the results, presented above, one must assume the existence of a layer of thickness $d$ at the illuminated side of the sample. This is the mechanically damaged layer, whose expanding and bending due to the absorption of the optical energy does not generate the photothermal piezoelectric signal. Therefore, the signal 
Fig. 5 Fit of the theoretical curve to the experimental piezoelectric spectrum of the etched $\mathrm{ZnSe}$ sample 2. Solid line theoretical curve, circles experimental data of sample 2 . Parameters: $l=0.11 \mathrm{~cm}$, $f=126 \mathrm{~Hz}, \alpha=0.05 \mathrm{~cm}^{2} \cdot \mathrm{s}^{-1}$, thickness of a damaged layer $d=0.014 \mathrm{~cm}$

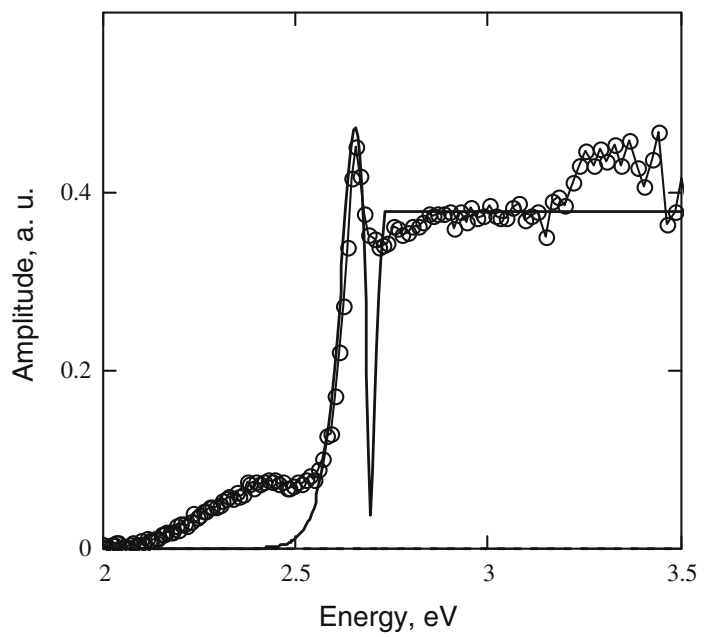

is generated in the remaining part of the sample. To simulate the piezoelectric amplitude and phase spectra, one can propose a modification of the J-A theory for the case of a mechanically damaged surface layer of the sample. Then the J-A equation takes the form of

$$
S \cong k\left(\frac{1}{l} \int_{d}^{l} T(x) \mathrm{d} x-\frac{6}{l^{2}} \int_{d}^{l}\left(\frac{l}{2}-x\right) T(x) \mathrm{d} x\right)
$$

Figure 2a presents a simulation of the amplitude piezoelectric spectra of the sample for a thermal diffusivity $\alpha=0.05 \mathrm{~cm}^{2} \cdot \mathrm{s}^{-1}$, thickness $l=0.11 \mathrm{~cm}$, frequency of the modulation $f=126 \mathrm{~Hz}$, and parameter $\gamma=0.99$ for three thicknesses of the damaged layer: $d=0.004 \mathrm{~cm}$ (solid), $d=0.013 \mathrm{~cm}$ (dots), and $d=0.02 \mathrm{~cm}$ (dash). The decrease of the signal in the high absorption region with an increase of the thickness of the damaged layer is clearly visible. Figure $2 b$ presents the simulation of the phase piezoelectric spectra computed for the same set of material and experimental parameters.

This model of a mechanically damaged layer was next used to interpret the experimental data of the ground, polished, and etched samples. The results of the fitting of the theoretical spectra to the experimental curves are presented in Figs. 3, 4, and 5. In these figures, the influence of the surface preparation on the piezoelectric photothermal spectra is clearly visible and expressed by the thickness of a damaged layer $d$.

For the ground sample spectra, the two maxima in the sub-bandgap region dominate, their intensity decreases after polishing. This is caused by the surface states. The ground samples exhibit also the smallest piezoelectric amplitude in the band-toband absorption region above the energy gap of the crystal. The peak at $E=2.65 \mathrm{eV}$ turned out to be caused by the absorption of light in the Urbach tail absorption region. The polishing improves the quality of the surface by reduction of the thickness of a mechanically damaged layer and a density of surface states. The etching process also 
Fig. 6 Piezoelectric (a) amplitude and (b) phase spectra of ZnSe crystal of thickness $l=0.11 \mathrm{~cm}$ cut and etched in $\mathrm{HCl}$ and measured at $f=$ $36 \mathrm{~Hz}$. Fitting parameters: $\alpha=0.05 \mathrm{~cm}^{2} \cdot \mathrm{s}^{-1}, E_{\mathrm{g}}=$ $2.71 \mathrm{eV}, \gamma=0.99$, $\beta_{o}=100 \mathrm{~cm}^{-1}, d=0 \mathrm{~cm}$ (dotted line), $d=0.0049 \mathrm{~cm}$ (dashed line), $d=0.02 \mathrm{~cm}$ (solid line). Circles are experimental points
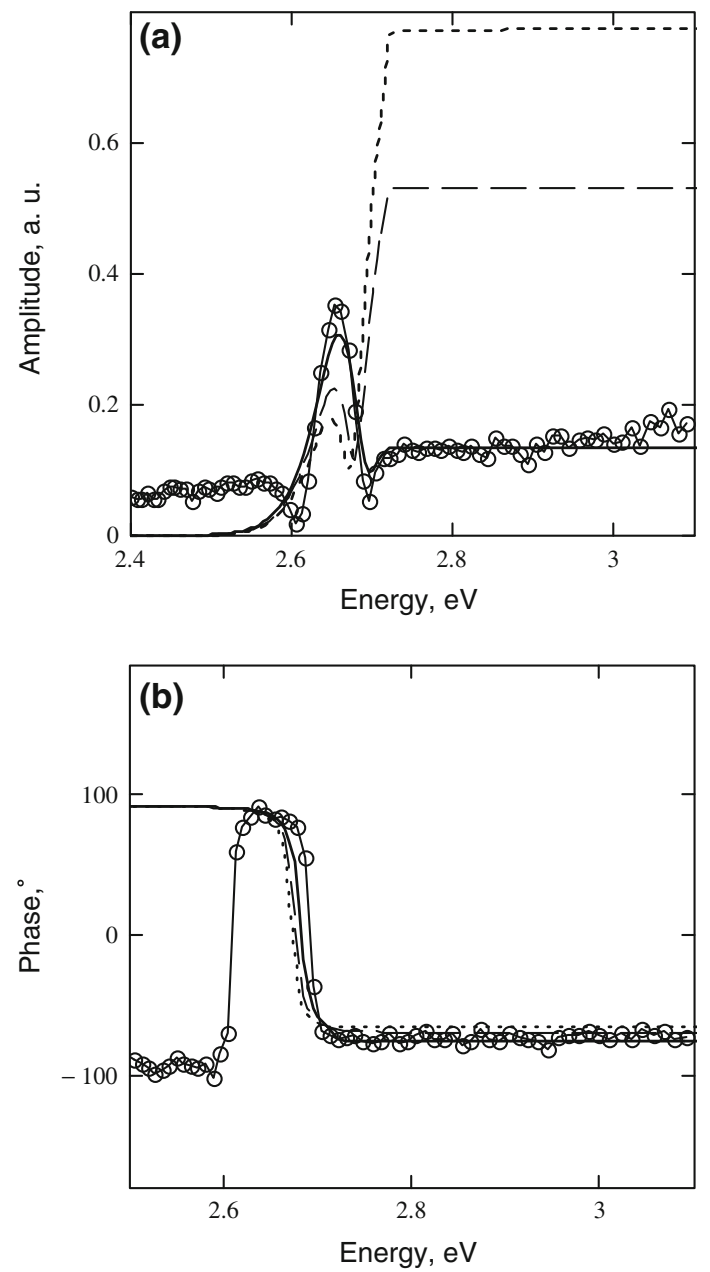

improves the quality of the surface by the constant decrease of the mechanically damaged layer. The improvement of the quality of the surface was expressed by a change of the thickness of the mechanically damaged layer $d$, decreasing from $d=0.018 \mathrm{~cm}$ to $d=0.016 \mathrm{~cm}$ and finally to $d=0.014 \mathrm{~cm}$ in the analyzed case of ZnSe samples.

Piezoelectric amplitude and phase spectra of $\mathrm{ZnSe}$ samples after cutting and etching in $\mathrm{HCl}$ and measured at the frequency $f=36 \mathrm{~Hz}$ are shown in Fig. 6. The above presented spectra, of unpolished samples, exhibit the existence of a thick mechanically destroyed layer of a thickness up to $d=0.02 \mathrm{~cm}$. The influence of the grinding of the same sample on the piezoelectric spectrum is shown in Fig. 7.

In this case a grinding process introduced larger destruction of the surface of the sample. 
Fig. 7 Piezoelectric (a) amplitude and (b) phase experimental and theoretical amplitude spectra of $\mathrm{ZnSe}$ cut, etched in $\mathrm{HCl}$ and next ground, measured at $f=36 \mathrm{~Hz}$. Fitting parameters: $\alpha=0.05 \mathrm{~cm}^{2} \cdot \mathrm{s}^{-1}$, $E_{\mathrm{g}}=2.71 \mathrm{eV}, \gamma=0.99$, $\beta_{\mathrm{o}}=100 \mathrm{~cm}^{-1}, d=0 \mathrm{~cm}$ (dotted line), $d=0.0049 \mathrm{~cm}$ (dashed line), $d=0.02 \mathrm{~cm}$ (solid line). Circles are experimental points
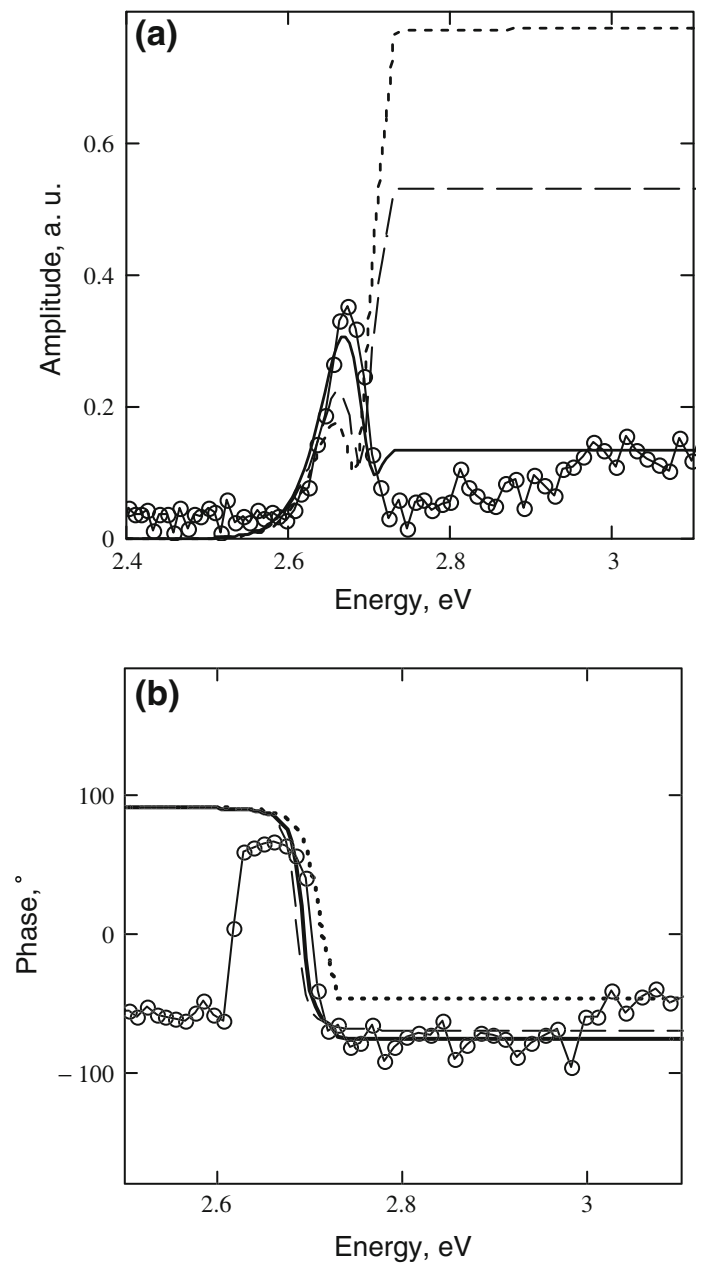

The theoretical dependence of the ratio of the amplitudes of the piezoelectric signals in the band-to-band and Urbach tail regions versus the thickness of a damaged layer is shown in Fig. 8.

\section{Conclusions}

The theoretical analysis of the piezoelectric experimental spectra, performed in the modified Jackson and Amer model, enabled an explanation of the origin of the peak at $E=2.65 \mathrm{eV}$ below the energy gap of the crystal located at $E=2.72 \mathrm{eV}$. Its nature is not related to the existence of donors or acceptors in the crystal but it is the result of the existence of the mechanically damaged layer, being the result of the grinding and polishing of the samples in the presence of the Urbach absorption tail below the energy gap of the crystal. It turned out that the piezoelectric method of detection is a 
Fig. 8 Ratio of the amplitudes of the piezoelectric signals in the band-to-band absorption region at $2.8 \mathrm{eV}$ and the Urbach tail region at $2.6 \mathrm{eV}$ as a function of the thickness of a damaged layer $d$. Parameters of computations: $f=127 \mathrm{~Hz}, \alpha=0.05 \mathrm{~cm}^{2} \cdot \mathrm{s}^{-1}$, $l=0.11 \mathrm{~cm}$

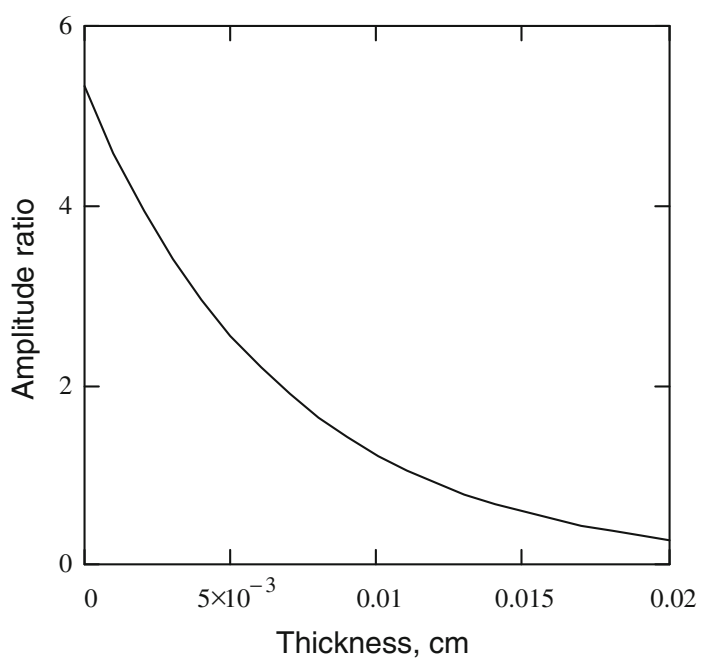

useful tool for estimation of the quality of the surface of semiconductors as it can be described by the thickness of the surface layer $d$. The studies presented in the paper showed also that the procedure of the surface treatment described in Sect. 2 composed of grinding, polishing, and etching of ZnSe samples still needs improvement. That improvement can be now checked by analysis of the piezoelectric spectra and determination of the thickness of a damaged layer $d$. Theoretical considerations show that for $\mathrm{ZnSe}$ samples the ratio of the amplitudes of the piezoelectric signal for the band-to-band absorption region to the amplitude of the signal for $E=2.65 \mathrm{eV}$ should be larger than 3 for $d=0.004 \mathrm{~cm}$ and 6 for perfectly prepared samples, i.e., for $d=0$ $\mathrm{cm}$. With the present method of surface treatment for ZnSe samples, described in the paper, it is still below 1 .

The thickness of the surface layer determined from the piezoelectric photothermal amplitude spectra for $\mathrm{Zn}_{1-x} \mathrm{Be}_{x}$ Se type mixed crystals is about two times smaller than for $\mathrm{ZnSe}$ and is equal to about $70 \mu \mathrm{m}$ for $x=0.05[11,14-16]$ for the same surface treatment. This agrees with the 1.6 times increase of the microhardness of $\mathrm{Zn}_{1-x} \mathrm{Be}_{x}$ Se crystal, from $1 \mathrm{GPa}$ to $1.6 \mathrm{GPa}$, with an increase of parameter $x$ from $x$ $=0$ to $x=0.05$ [17]. Observed changes of the thickness of the damaged layers after polishing $30 \mu \mathrm{m}$ and etching $20 \mu \mathrm{m}$ were confirmed by corresponding measurements of the thickness of the samples.

Open Access This article is distributed under the terms of the Creative Commons Attribution License which permits any use, distribution, and reproduction in any medium, provided the original author(s) and the source are credited.

\section{References}

1. D.P. Almond, P.M Patelm, Photothermal Science and Techniques (Chapman \& Hall, London, 1996)

2. A. Fukuyama, S. Sakamoto, S. Sonoda, P. Wang, K. Sakai, T. Ikari, Thin Solid Films 112, 511 (2006)

3. Q. Shen, K. Abe, T.I. Shigenari, T. Toyoda, J. Lumin. 87-89, 444 (2000) 
4. Y. Song, D.M. Todorovic, B. Cretin, P. Vairac, Int. J. Solids Struct. 47, 1871 (2010)

5. K. Yoshino, H. Mikami, M. Yoneta, T. Ikari, J. Lumin. 87-89, 608 (2000)

6. P. Wang, T. Nakagawa, A. Fukuyama, K. Maeda, Y. Iwasa, M. Ozeki, Y. Akashi, T. Ikari, Mater. Sci. Eng. C 26, 826 (2006)

7. K. Yoshino, Y. Nakagawa, A. Fukoyama, H. Yokoyama, K. Maeda, H. Ishikura, T. Abe, T. Ikari, Phys. Status Solidi B 210, 491 (1998)

8. W. Jackson, N.M. Amer, J. Appl. Phys. 51, 3343 (1980)

9. I.V. Blonskij, V.A. Thoryk, M.L. Shendeleva, J. Appl. Phys. 79, 3512 (1996)

10. M. Maliński, J. Zakrzewski, K. Strzałkowski, Int. J. Thermophys. 28, 299 (2007)

11. M. Maliński, J. Zakrzewski, K. Strzałkowski, S. Łęgowski, F. Firszt, H. Męczyńska, Surf. Sci. 603, 131 (2009)

12. M. Maliński, L. Bychto, J. Zakrzewski, J. Phys. IV 129, 245 (2005)

13. J. Zakrzewski, M. Maliński, K. Strzałkowski, F. Firszt, S. Łęgowski, H. Męczyńska, A. Marasek, M. Pawlak, J. Phys. IV 137, 381 (2006)

14. K. Yoshino, H. Mikami, K. Imai, M. Yoneta, T. Ikari, Physica B 302-303, 299 (2001)

15. F. Firszt, J. Zakrzewski, K. Strzałkowski, M. Maliński, S. Łęgowski, H. Męczyńska, A. Marasek, Y.S. Huang, D. Dumcenco, Phys. Status Solidi C 7, 1463 (2010)

16. F. Firszt, K. Strzałkowski, J. Zakrzewski, S. Łęgowski, H. Męczyńska, M. Maliński, D. Dumcenco, Ch.T. Huang, Y.S. Huang, Phys. Status Solidi B 247, 1402 (2010)

17. W. Paszkowicz, K. Godwod, J. Domagała, F. Firszt, J. Szatkowski, H. Męczyńska, S. Łęgowski, M. Marczak, Solid State Commun. 102, 735 (1998) 